\title{
Multiple Spinal Intradural-Intramedullary Involvement by Metastatic Carcinoma with Neuroendocrine Differentiation with Occult Primary-An Unusual Case Report and Review of Literature
}

\author{
Anshu Gupta1*, Sachin Sinha² \\ ${ }^{1}$ Pathology, Institute of Human Behavior and Allied Sciences (IHBAS), Delhi, India \\ ${ }^{2}$ Oral Pathology, Narain Sewa Sansthan, Bengaluru, India \\ Email: *dransh2002@yahoo.co.in,drsachinsinha@rediffmail.com
}

Received 13 November 2015; accepted 17 April 2016; published 20 April 2016

Copyright (C) 2016 by authors and Scientific Research Publishing Inc.

This work is licensed under the Creative Commons Attribution International License (CC BY).

http://creativecommons.org/licenses/by/4.0/

(c) (i) Open Access

\begin{abstract}
Although vertebral column is recognized as the most common site for bony metastasis in patient with systemic malignancy, intramedullary metastases to the spinal cord is infrequent. Between $5 \%$ - $10 \%$ of cancer patients develop spinal metastasis during the course of their diseases. Intramedullary tumors are rare, comprising $3.5 \%$ of spinal metastasis. Most metastatic spinal lesions (70\%) are found at the thoracic level, $20 \%$ in lumbar region and $10 \%$ in the cervical region. We report a rare biopsy proven case of intramedullary spinal metastatic carcinoma with neuroendocrine differentiation because of its unusual presentation, involving spine at multiple noncontiguous levels, which appeared as irregular small nodules on MRI. The primary tumor was most likely from occult primary in lung. Biopsy from the spinal lesion established the diagnosis of metastatic carcinoma with neuroendocrine differentiation. Therefore, in patients with spinal metastasis, a thorough work up is advised to evaluate primary site. This would help to delineate the nature and the extent of the systemic disease. We highlighted herein the clinical presentation, radiological findings particularly MRI and role of biopsy in the diagnosis and treatment of intramedullary spinal metastasis.
\end{abstract}

Keywords

Spinal Cord, Metastasis, Carcinoma, Neuroendocrine

\footnotetext{
${ }^{*}$ Corresponding author.
}

How to cite this paper: Gupta, A. and Sinha, S. (2016) Multiple Spinal Intradural-Intramedullary Involvement by Metastatic Carcinoma with Neuroendocrine Differentiation with Occult Primary-An Unusual Case Report and Review of Literature. Open Journal of Pathology, 6, 105-110. http://dx.doi.org/10.4236/ojpathology.2016.62013 


\section{Introduction}

Intramedullary spinal cord metastases (ISCMs) are an unusual complication of malignancies outside the central nervous system [1]. Intramedullary tumors arise within the substance of the spinal cord whereas extramedullary tumors are extrinsic to the cord [2]. Metastatic lesions occur at multiple noncontiguous levels in $10 \%$ to $38 \%$ of cases. As many as $10 \%$ of the patients with symptomatic spinal metastases present with no known primary lesion [3]. Intramedullary metastasis arises hematogenously from tumor emboli in para-vertebral venous plexus, which is characterized by absence of valves or from direct extension from the nerve roots or CSF. The clinical presentation of the metastatic spinal disease is predominantly pain, neurological deficit, progressive deformity, general weakness and bladder and bowel dysfunction. MRI is now the method of choice to detect the presence and extent of spinal metastasis. It provides excellent visualization of spinal cord involvment, ligamentous involvment, spinal cord edema, and degree of canal compromise and cord compression. Biopsy from the affected site proves to be useful in the confirmative diagnosis, treatment and prognosis of metastatic lesion. Prognosis is based on degree of deficit, duration of symptoms, type and location of tumor and degree of advancement of disease. Management of metastatic spinal tumor is focused on pain relief, preservation of neurologic function, prevention of pathologic fracture and correction of spinal instability for improving quality of life. Palliation is the real treatment goal. Treatment includes conservative and operative treatment. Conservative treatment methods are analgesics and braces, chemotherapy, radiotherapy and steroid therapy.

\section{Case Presentation}

A 38 year old male presented with progressive weakness in bilateral lower limbs from 2 - 3 months. Patient also develops urinary incontinence in the due course of time and finally patient became bed-ridden. Patient had no history of cough or respiratory distress or pain abdomen. On examination, no thyroid swelling was palpable on deglutination. Per abdomen and pelvic examination were normal. Routine investigations that included hematological and biochemical ( $\mathrm{T}_{3}, \mathrm{~T}_{4}, \mathrm{TSH}$, urea, creatinine etc.) investigations were normal. X-ray and CT chest were also normal. Ultrasound abdomen and pelvis showed no mass or any other abnormal finding. MRI of whole spine done with following findings: MRI dorsolumbar spine revealed nodular intradural and intramedullary lesions in dorsal aspect of cord at T1, T5 and T10 level producing intramedullary T2 hyperintense signal intensity from C7 to T2 and T9 to conus. Mild diffuse disc bulge is seen at the level of C3/C4, C4/C5 and C6/C7 (Figure 1). MRI Lumbosacral spine revealed intramedullary T1 hypointense signal intensity in the conus associated with T1/T2 hypointense loculated cystic lesion in the spinal canal at S1 \& S2 level (Figure 2). A tiny nodular intermediate signal intensity lesion was seen with cauda equine nerve root at L4 nerve root. Cerebrum, cerebellum and brain stem showed no significant pathology. The lesions in lumbo-sacral region resulted in clinical symptoms of weakness in bilateral lower limbs and urinary incontinence.

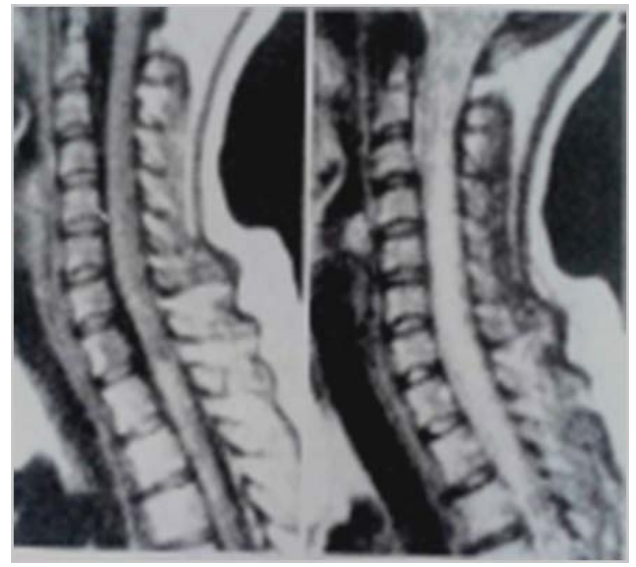

(a) (b)

Figure 1. (a) \& (b) MRI cervico-thoracic region showing intramedullary lesions producing $\mathrm{T}_{1}$ hypointense $/ \mathrm{T}_{2}$ hyperintense signal intensity from $\mathrm{C} 7$ to $\mathrm{D} 2$ respectively. 


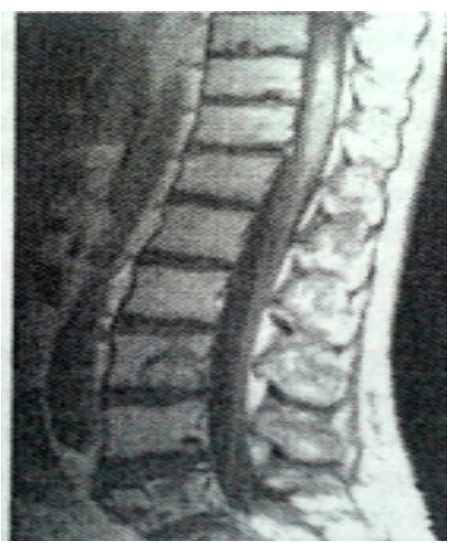

Figure 2. MRI lumbo-sacral region showing intramedullary heterogeneously enhancing masses in the lumbar \& sacral region in $\mathrm{T}_{1}$.

Intra-operative biopsy from nodular lesion in dorsolumbar spine was performed and sent to pathology department for frozen section examination. A provisional diagnosis of malignant small round cell tumor was made on frozen section by cryostat. Based on the provisional diagnosis, further excision biopsy done to relieve compression symptoms and sent for microscopic examination. The biopsy specimen showed multiple grayish brown soft tissue pieces that together measured $2.8 \times 2.5 \times 0.5 \mathrm{cms}$ grossly. Cut surface was gray white with few hemorrhagic areas. Paraffin sections were prepared and stained with routine Haematoxylin and Eosin (H \& E) stain, special stains such as reticulin and immunohistochemical stains for cytokeratin, synaptophysin and chromogranin.

\subsection{Procedure for Haematoxylin and Eosin (H \& E) Stain}

1). Take sections to water.

2). Place sections in haematoxylin for 5 minutes.

3). Wash in tap water.

4). "Blue" sections in Scott's tap water.

5). Wash in tap water.

6). Place sections in $0.3 \%$ acid alcohol for a few seconds.

7). Wash in tap water.

8). Place sections in eosin for 2 minutes.

9). Wash in tap water.

10). Dehydrate, clear and mount sections in DPX.

Results

Nuclei-blue-black, cytoplasm-varying shades of pink, muscle fibres-deep pinky red, fibrin-deep pink, red blood cells-orange/red.

\subsection{Immunohistochemical (IHC) Staining Procedure}

1). Paraffin embedded 4 micron-thick sections used.

2). Dewax in xylene, 3 - 5 min

3). Place the slides into glass slide chamber and fill them with the processing buffer (citric acid buffer, ph 6.04 .

4). Antigen retrieval by autoclave $\left(121^{\circ} \mathrm{C}, 15 \mathrm{~min}\right)$.

5). Take out the glass slides in chamber, wait for $40 \mathrm{~min}$, and rinse the slides 3 times with distilled water or Phosphate Buffer Solution (PBS) by emptying and refilling the chambers.

6). Block endogenous peroxidase activity with freshly made $0.3 \% \mathrm{H}_{2} \mathrm{O}_{2}$ in methanol, 20 min.

7). Three changes of PBS for five minutes.

8). Incubate with monoclonal primary antibody, $4^{\circ} \mathrm{C}$, overnight.

9). Wash with PBS, $3 \times 5$ min. 
10). Incubate with secondary antibody, 60 - $120 \mathrm{~min}$. at room temperature.

11). Three changes of PBS for five minutes.

12). Stain with diaminobenzidin (DAB) solution, $10 \mathrm{~min}$. at room temperature. $0.01 \% \mathrm{DAB}$ in $0.5 \mathrm{M}$ Tris/ $\mathrm{HCI}\left(\mathrm{pH}\right.$ 7.4) solution should be filtrated. $\mathrm{H}_{2} \mathrm{O}_{2}$ must be added to a final concentration of $0.01 \%$.

13). Wash with running tap water, 3 min.

14). Counterstain with Mayer's hematoxylin, 30 sec.

15). Wash with running tap water.

16). Dehydrate with increasing solutions of ethanol: 50\%, 70\%, 96\%, absolute, 3 min. each.

17). Clear with xylene, $3 \times 3$ min.

18). Mount with mounting medium.

Microscopic examination: Biopsy showed a cellular tumor in which cells were arranged in sheets, well defined clusters, trabeculae, around blood vessels forming pseudo-rosettes and scattered diffusely in highly vascularised fibrocollagenous stroma. Tumor cells were small, monomorphic round to oval to elongated with moderate atypia, stippled chromatin and inconspicous nucleolus. Cytoplasm is scanty and barely visible. Nuclear molding was seen in occasional cells. Some of the cells were slightly larger and showed moderate amount of eosinophilic cytoplasm. Mitosis was infrequent (Figure 3). Reticulin stain showed reticular fibers around group of cells. On immunohistochemistry, tumor cells showed diffuse positivity for cytokeratin, synaptophysin and chromogranin (Figure 4). S-100 was focally positive. Vimentin positivity is seen in fibro connective stroma. Findings were that of metastatic carcinoma with neuroendocrine differentiation. Small cell carcinoma lung was considered as the first possibility but chest X-ray and CT lung was normal.

Postoperatively, patient was given external beam radiotherapy but continued to experience pain and paraesthesias. Patient showed no improvement and his general condition gradually deteriorated in due course of time. Ultimately, he died within six months.

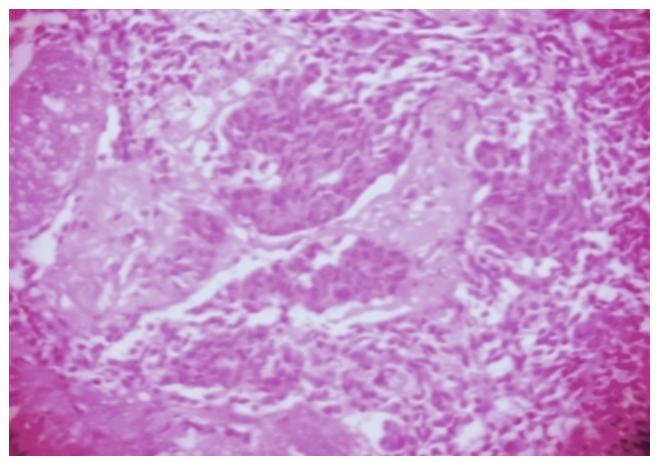

Figure 3. Tumor cells in clusters and scattered diffusely in loosely vascularised fibrocollagenous stroma. (Haematoxylin \& Eosin stain, 10×).

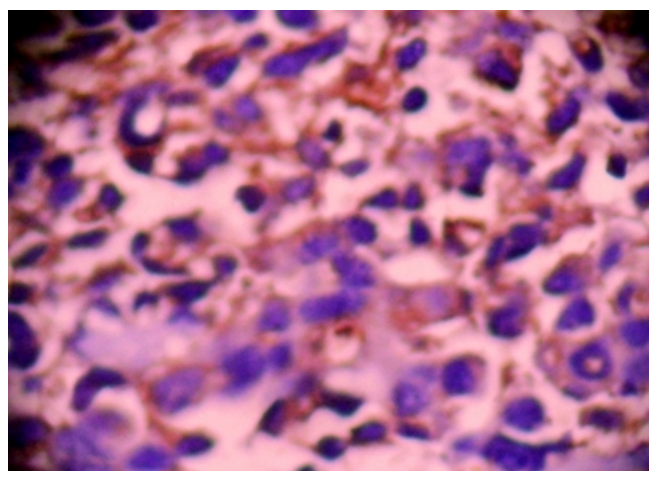

Figure 4. Microphotograph showing diffuse positivity (brown) for synaptophysin in cytoplasm of tumor cells (Immunohistochemistry, 40×). 


\section{Discussion}

Spinal metastases occur 20 times more commonly than primary tumors of spine. Five to ten percent of cancer patients develop spinal metastasis [3]. Spinal metastasis are often multiple and are frequently seen late in the course of disease, in which brain or visceral metastasis are also evident. Intramedullary spinal cord metastases (ISCMs) represent only $0.9 \%$ to $2.1 \%$ of autopsy cases in patients with cancer [4]. One third of adult spinal cord tumors are intramedullary and rest are extramedullary. Of the tumors metastasizing to the spinal cord lung carcinoma is the most common accounting for $50 \%$ followed by breast carcinoma, Lymphoma, melanoma, colorectal carcinoma, Hodgkin disease, head and neck carcinoma and leukemia [5] [6]. Primary tumors less commonly reported to metastasize to spine include schwannoma, mesothelioma, merkel tumor, plasmacytoma, teratoma, as well as basal cell, parotid, nasopharyngeal, laryngeal, esophageal, gall bladder, pancreas, ovarian, endometrial, and urinary bladder tumors. Only about $2 \%$ of spinal metastasis is of unknown origin [7].

There is a slight preponderance of metastatic spinal tumors in males (60\%) compared to females. In our case, the patient was a male of 38 years of age.

Grossly, the spinal cord appears firm and swollen, frequently in a fusiform manner [7]. Multiple segments are often affected. Metastatic lesions occur at multiple noncontiguous levels in $10 \%$ to $38 \%$ of cases [8]. Leptomeningeal and intradural involvement can be found in conjunction with the intramedullary tumor [9] [10]. In our case, there was intradural and intramedullary involvement of entire spinal cord at multiple levels causing nodular enlargement with no evidence of any lesion in brain or primary in lung, thyroid, pancreas, stomach, colon, prostate etc.

Extradural metastases account for approximately $95 \%$ of secondary spinal tumors. Intradural extramedullary metastases are uncommon. Intramedullary tumors are rare, comprising $3.5 \%$ of spinal metastasis. Intramedullary metastasis arises hematogenously from tumor emboli followed by para-vertebral venous plexus, which is characterized by absence of valves or from direct extension from the nerve roots or CSF [11].

Patients with spinal cord metastases arising from primary tumors outside the CNS show rapid onset of symptoms. The clinical features of the metastatic spinal disease are predominantly pain, neurological deficit, progressive deformity, general weakness and bladder and bowel dysfunction as in our case. It is axiomatic that new onset back or neck pain in a cancer patient means spinal metastasis until proved otherwise [12].

In the diagnostic work up, plain radiograph can show spinal alignment, the presence of fracture, and gross involvment by tumor. MRI is now the method of choice to detect the presence and extent of spinal metastasis. MRI imaging proves helpful because multiple lesions that are not suspected clinically are found in $30 \%$ of cases [13] [14]. In our case, MRI findings revealed intramedullary T2 hyperintense signal intensity from C7 to T2 and D9 to conus.

When primary cancer is not yet identified, metastatic extension can be difficult to detect [12]. In our case, primary site could not be ascertained despite all investigations and detailed examination of the patient, which remained the main limitation of our study.

Biopsy from the affected site proves to be useful in the confirmative diagnosis, treatment and prognosis of metastatic lesion. A direct biopsy during surgery prior to definitive surgery is advantageous for the patients [15]. In our case biopsy from the nodular lesion was reported as metastatic carcinoma with neuroendocrine differentiation based on routine $\mathrm{H} \& \mathrm{E}$ staining and further confirmed by immunohistochemistry which showed positivity for synaptophysin, chromogranin and cytokeratin. Small cell carcinoma lung was considered in differential diagnosis but could not get supportive clinical and radiological findings to make a definitive diagnosis.

Management of metastatic spinal tumor should be focused on pain relief, preservation of neurologic function, prevention of pathologic fracture and correction of spinal instability for improving quality of life. Treatment includes conservative and operative treatment. Conservative treatment methods are analgesics and braces, chemotherapy, radiotherapy and steroid therapy. Rapidly progressive neurological deterioration can not be recovered by radiotherapy because immediate spinal decompression is not possible.

Prognosis is uniformly poor with life expectancy of months as this represents an advanced stage of disease [6]. Prognosis is based on degree of deficit, duration of symptoms, type and location of tumor and degree of advancement of disease. Our patient had poor prognosis and died within few months due to rapid disease progression [15].

\section{Conclusion}

This report pertains to a rare case of intramedullary spinal metastatic carcinoma at multiple sites with unknown 
primary having poor prognosis. Therefore, in patients with spinal metastasis, a thorough work up is advised to evaluate primary site. This would help to delineate the nature and the extent of the systemic disease. Treatment and prognosis depends upon age of the patient, site and extent of involvement in spinal cord, alignment of spine and histological typing of the tumor. Depending on this, various modalities such as surgery, radiotherapy and chemotherapy are provided to improve quality of life and prognosis. Loss of sphincter control is a poor prognostic feature and is mostly irreversible. This report is of interest to multiple specialities. No treatment has been proven to increase the life expectancy of patients with lung cancer and spinal metastasis. Pain relief and maintenance of quality of life must be balanced against the patient life expectancy, presence of co-morbidities, immunological, nutritional and functional status.

\section{Consent}

A written informed consent was obtained from the patient's next kin for the publication of this case report and the accompanying images.

\section{References}

[1] Edelson, R.N., Deck, M.D.F. and Posner, J.B. (1972) Intramedullary Spinal Cord Metastases. Clinical and Radiographic Findings in Nine Cases. Neurology, 22, 1222-1231. http://dx.doi.org/10.1212/WNL.22.12.1222

[2] Scubba, D.M. and Gokasla, Z.L. (2006) Diagnosis and Management of Metastatic Spine Disease. Surgical Oncology, 15, 141-151. http://dx.doi.org/10.1016/j.suronc.2006.11.002c

[3] Botterell, E.H. and Eitzgerald, G.N. (1959) Spinal Compression Produced by Extradural Malignant Tumors. Canadian Medical Association Journal, 80, 791-796.

[4] Kalayci, M., Cagavi, F., Gul, S., Yendunya, S. and Acikgoz, B. (2004) Intramedullary Spinal Cord Metastases: Diagnosis and Treatment-An Illustrated Review. Acta Neurochir (Wein), 146, 1347-1354. http://dx.doi.org/10.1007/s00701-004-0386-1

[5] Smaltino, F., Bernini, F.P. and Santoro, S. (1980) Computerized Tomography in the Diagnosis of Intramedullary Metastases. Acta Neutochir (Wein), 52, 299-303. http://dx.doi.org/10.1007/BF01402085

[6] Jellinger, K., Kothbauer, P., Sunder-Plassmann, E. and Weiss, R. (1979) Intramedullary Spinal Cord Metastases. Journal of Neurology, 202, 31-41. http://dx.doi.org/10.1007/BF00313146

[7] Grem, J.L., Burgess, J. and Trump, D.L. (1985) Clinical Features and Natural History of Intramedullary Spinal Cord Metastasis. Cancer, 56, 2305-2314. http://dx.doi.org/10.1002/1097-0142(19851101)56:9<2305::AID-CNCR2820560928>3.0.CO;2-X

[8] Hirose, G., Shimazaki, K., Takado, M., Kosoegawa, H., Ohya, N. and Mukawa, A. (1980) Intramedullary Spinal Cord Metastasis Associated with Pencil-Shaped Softening of the Spinal Cord. Journal of Neurosurgery, 52, 718-721. http://dx.doi.org/10.3171/jns.1980.52.5.0718

[9] Guyer, P.B., Westbury, H. and Cook, P.L. (1968) The Myelographic Appearances of Spinal Cord Metastases. The British Journal of Radiology, 41, 615-619. http://dx.doi.org/10.1259/0007-1285-41-488-615

[10] Costigan, D.A. and Winkelman, M.D. (1985) Intramedullary Spinal Cord Metastasis. A Clinicopathological Study of 13 Cases. Journal of Neurosurgery, 62, 227-233. http://dx.doi.org/10.3171/jns.1985.62.2.0227

[11] Wood, E.H., Taveras, J.M. and Pool, J.L. (1953) Myelographic Demonstration of Spinal Cord Metastases from Primary Brain Tumors. American Journal of Rontgenology, 69, 221-230.

[12] Benson, D.F. (1960) Intramedullary Spinal Cord Metastases. Neurology, 10, 281-287. http://dx.doi.org/10.1212/WNL.10.3.281

[13] Cuénod, C.A., Laredo, J.D., Chevret, S., et al. (1996) Acute Vertebral Collapse Due to Osteoporosis or Malignancy: Appearance on Unenhanced and Gadolinium-Enhanced MR Images. Radiology, 199, 541-549. http://dx.doi.org/10.1148/radiology.199.2.8668809

[14] Greenberg, A.D., Scatliff, J.H., Selker, R.G. and Marshall, M.D. (1965) Spinal Cord Merastasis from Bronchogenic Carcinoma. A Case Report. Journal of Neurosurgery, 23, 72-75. http://dx.doi.org/10.3171/jns.1965.23.1.0072

[15] Lee, C.S. and Jung, C.H. (2012) Metastatic Spinal Tumor. Asian Spine Journal, 6, 71-87. 